\title{
Role of Diffusion-Weighted MR Imaging in Characterization of Posterior Fossa Tumors
}

Lubna Ali Hussain (DMRD) ${ }^{*}$ Noor Abbas Hummadi(FABMS-radiodiagnosis) ${ }^{* *}$, Nibras Jassam Homadi (FIBMSadiodiagnosis) ${ }^{*}$, Wasan Ismail AI-Saadi(FIBMS-radiodiagnosis) ${ }^{* *}$

\begin{abstract}
Background: Conventional MR imaging is essential for diagnosis and evaluation of the posterior fossa tumors Objectives: To assess the value of diffusion weighted imaging and apparent diffusion coefficient in making distinction between different histological types of posterior fossa tumors.
\end{abstract}

Type of the study: Cross-sectional study.

Methods: Brain MRI and DWI assessed 19 patients (12 female and 7 male) with MRI diagnosis of posterior fossa tumors. absolute ADC values of contrast -enhancing solid tumor region and $A D C$ ratio of solid tumor to $A D C$ of normal -appearing deep White matter were compared with histological diagnosis postoperatively .The mean ADC value and ratio were determined by using a 2 -tailed $T$ test. Results: In eight of medulloblastoma ,The mean ADC value was $0.67 \pm 0.14 \times 10^{-3} \mathrm{~mm}^{2} / \mathrm{s}$ and ratio $0.86 \pm 0.19$, ependymoma $(n=3)$, ADC value $1.09 \pm 0.14 \times 10^{-3} \mathrm{~mm}^{2} / \mathrm{s}$ and ratio $1,43 \pm 0.13$, pilocytic astrocytoma $(n=4)$ ADC value $1,72 \pm 0.27 \times 10^{-3} \mathrm{~mm}^{2} / \mathrm{s}$ and ratio $2.19 \pm 0.4$, brainstem glioma $(n=2)$ with $A D C$ value1.18 $\pm 0.06 \times 10^{-}$ ${ }^{3} \mathrm{~mm}^{2} / \mathrm{s}$ and ratio $1.64 \pm 0.08$, haemangioblastoma $(\mathrm{n}=1)$ with $A D C$ value $1.46 \times 10^{-1} \mathrm{~mm}^{2} / \mathrm{s}$ and ratio 1.87 , and meningioma with $A D C$ value $0.72 \times 10^{-3} \mathrm{~mm}^{2} / \mathrm{s}$ and ratio 0.85. ADC values were higher in JPA than in ependymomas and medulloblastoma ( $P$ value
$<0,05)$.ADC ratio were also statistically significant difference among these three tumors type .

Conclusion: calculation of apparent diffusion coefficient value in the solid enhancing portion of tumor seems to be reliable for differentiate between medulloblastoma at one end and, juvenile pilocytic astrocytoma (JPA) and ependymoma at other end as the former has ADC of $<1$ while other tumors have ADC value of $>1$.

Key words : posterior fossa tumors, DWI ,ADC

\section{Al-Kindy College Medical Journal 2018: Vol. 14 No. 1} Page: 8-12

\author{
${ }^{*}$ Radiology department / Al-Emamain Al-Kadhimain \\ Medical City \\ ** Surgery department / Al-Nahrain College of \\ Medicine
}

Received $26^{\text {th }}$ Aug 2016, accepted in final $12^{\text {th }}$ March 2017

Corresponding to Nibras Jassam Homadi, e-mail address : nibrasjassam@yahoo.co.uk
$\mathrm{C}$ onventional MR imaging is essential for diagnosis and evaluation of the posterior fossa tumors, however the histological type \& grading can be accurately predicted in only $70 \%$ of cases (1)The posterior fossa is the most common site for intracranial neoplasms in the pediatric population. Medulloblastoma and cerebellar astrocytoma account for about two thirds of all posterior fossa neoplasms in children, with ependymoma and brainstem glioma composing the remaining third. The hemangio blastomais common primary cerebellar neoplasm in the adult population.(2,3)Accurate preoperative diagnosis is an important goal in pediatric patients with cerebellar neoplasms, because the most common tumors in this location and age group, juvenile pilocytic astrocytoma (JPA) and medulloblastoma, may dictate the need for different surgical approaches and have significantly different natural histories and outcomes.( 4)Diffusion -weighted MR imaging exploits the phenomenon of diffusion , which is related to Brownian motion at the molecular level. DWI takes advantage of the fact that intracellular water- molecules are much more limited in their movement than extracellular ones, because they quickly bump into the cell membrane that contains them.The more restricted the movement of water, the brighter it will be on DWI sequence.(5) Diffusion MR with calculated apparent diffusion coefficient (ADC) maps which represent an absolute measure of average diffusion for each voxel.(6) allow evaluation of microscopic water diffusion within tissues, Diffusion-weighted imaging (DWI) and ADC maps are useful in evaluation of acute infarcts and a number of different brain lesions.( 6 -9) ADC values in brain tumors seem to be primarily based on tumor cellularity and nuclear area, (10 -13) and a correlation between ADC values and tumor grade also seems to be present, though the reported studies have demonstrated conflicting results. (9 20) Significant differences in cellularity of pediatric 
cerebellar neoplasms, particularly between JPAs and medulloblastomas, indicate that these lesions could potentially be distinguished by their ADC values.( 12.21) The purpose of this study is To assess the value of diffusion weighted imaging and apparent diffusion coefficient in making distinction between the different histological types of posterior fossa tumors.

\section{Methods:}

Patients: This is a Prospective study conducted at MRI unit at AL-Imammain AL-Kadhimain medical city From December2014 through August 2015.the study was approved by the Ethics Committee of the Faculty of Medicine- Al Nahrain University and informed consent was obtained the study included 19 Patients with newly diagnosed posterior fossa tumor for whom conventional \& DWI MRI was performed \&the type of the lesion was ascertained by histopathology postoperatively. Exclusion criteria were Multiple posterior fossa lesions, Cystic lesion with no or small solid component less than $1 \mathrm{~cm}$ in largest dimension (for solid part), Previous posterior fossa surgery or irradiation and Previous cranial irradiation.

methods: MRI studies were performed using a $1.5 \mathrm{~T}$ whole - body MRI system Avanto, Siemens medical system, Germany and 1.5 T Achieva Philips medical system, Holland equipped with performance Gradients and manufacturer - supplied quadrature head coil. The standard imaging protocol consisted of the following sequences: non contrast T1WI, in axial (TR 500/ TE 9.7) and sagittal planes (TR 232 /TE4.76 ), coronal (TR232/ TE 4.76 ), fast spin echo T2WI axial (TR 5730/TE115), axial fluid attenuated inversion recovery FLAIR (TR8100/TE102) and post contrast axial, coronal and sagittal T1WIs . diffusion weighted MR imaging (DWI) : before the contrast enhanced imaging, routine diffusion - weighted MR imaging were obtained using single - shot multislice spin -echo EPI sequence (TR 5327, TE 162 ), and (TR 3700, TE141) with diffusion sensitivities of $b$ values $=1000 \mathrm{~s} / \mathrm{mm} 2$. the diffusion gradient were applied sequentially in three direction ( $\mathrm{X}, \mathrm{Y}, \mathrm{Z}$ direction ). total acquisition time was $80 \mathrm{~s}$. After the administration of $0.1 \mathrm{mmol} / \mathrm{kg}$ gadopentetate dimeglumine contrast enhanced T1 axial (TR 500/TE9.7), sagittal (TR 232/TE4.76) coronal ( TR 232 /TE4.76) images were obtained in all sequence, the field of view was 22 or $24 \mathrm{~cm}$ and section thickness was $5 \mathrm{~mm}$ with an inter slice gap of $1 \mathrm{~mm}$.

image analysis : DW images visually inspected and classified as hyper intense, isointense, or hypointense compared with normal white matter; The ADC map as obtained automatically for $b$-value (1000s/mm2) with a pixel -by pixel analysis, by using advanced work station software after DWI data acquisition. measurement of ADC value for maximally enhancing solid portion of the lesion ,avoiding the cystic and necrotic areas and for the contralateral white matter normal area by drawing the circular region of interest (ROIs) manually on all axial ADC map using the manufacturers software , the ROls measurements varied from $40-80 \mathrm{~mm} 2$ for tumor. $5-10 \mathrm{~mm} 2$ for the contralateral deep white matter, the ADC value were expressed in 10 . $3 \mathrm{~mm} 2 / \mathrm{s}$. ADC of the tumor were divided by the ADC of the normal white matter to find the normalized ADC (NADC) ratio. Histological diagnosis was provided by examination of post operative specimens and the correlated with $A D C$ value .

Statistical study: T-test was conducted calculating the difference in the mean $A D C$ value and the mean $A D C$ ratio between different posterior fosse tumors $\mathrm{P}<0.05$ was considered statistically significant .

Results: This prospective study included (19) patient (7) females (12) males. with age range from 1.5 -70 year. According to histopathology (8)patients had medulloblastoma,(3)patients had ependymoma, (4)patient had pilocystic astrocytoma ,(2) patients had brainstem glioma,(1)patient had hemangioblastoma,(1)patient had meningioma. The age distribution with respect to histopathology shown in table (1)

Table (1): Age distribution of patients according to type of tumors

\begin{tabular}{|c|c|c|c|}
\hline Histopathology & No. & $\begin{array}{c}\text { Rang } \\
\text { e yr }\end{array}$ & Mean \pm SDyr \\
\hline Medullablastoma & 8 & $\begin{array}{c}1.6- \\
26\end{array}$ & $10.45 \pm 8.66$ \\
\hline $\begin{array}{c}\text { Pilocytic } \\
\text { astrocytoma }\end{array}$ & 4 & $4-15$ & $9.75 \pm 5.56$ \\
\hline Ependymoma & 3 & $5-9$ & $6.67 \pm 2.08$ \\
\hline Brainstem Glioma & 2 & & $6.0 \pm 2.83$ \\
\hline Hemangioblastoma & 1 & & 16 \\
\hline Atypical Meningioma & 1 & & 70 \\
\hline
\end{tabular}

The ADC value and ratio for all the tumors are shown in table (2). The highest ADC value were recorded for pilocytic astrocytoma which was $(1.72 \pm 0.27 \times 10 \quad-3 \mathrm{~mm} \quad 2 \quad)$ followed by hemangioblastoma the ( $1.46 \times 10-3 \mathrm{~mm} 2)$,brainstem glioma( $1.18 \pm 0.06 \quad \times 10-3 \mathrm{~mm} 2$ )ependymoma(1.09 $\pm 0.1 .4 \times 10-3 \mathrm{~mm} 2)$ and atypical meningioma $(0.72 \times 10-3 \mathrm{~mm} 2)$. The least ADC 
value was reported for medulloblastoma $(0.67 \pm 0.14 \times 10-3 \mathrm{~mm} 2)$ table (2).Fig(1) The highest $A D C$ ratio recorded for pilocytic astrocytoma $2.19 \pm 0.4$, brainstem glioma $1.64 \pm 0.08$, followed by ependymoma $\quad 1.43 \pm 0.13$, hemangioblastoma 1.87 ,atypical meningioma 0.85 , and least $A D C$ ratio were reported for medulloblastoma $0.86 \pm 0.19 \mathrm{Fig}(2)$ In cases of medulloblastoma( $n=8)$, the diffusion was significantly restricted in comparison with JPA and ependymoma in $p<0.05$. All of them appeared predominantly hyper intense on DWI relative to normal brain parenchyma, hypo intense on ADC map and had $A D C$ value ranged between $0.67 \pm 0.14$ $x \quad 10-3 \mathrm{~mm} 2 / \mathrm{s}$ and ADC ratio $0.86 \pm 0.19$ table (2) and which is consist with marked restriction of diffusion .Figure (1)

Table (2): Data of ADC value and Ratio of different posterior fossa tumors

\begin{tabular}{|c|c|c|}
\hline Histopathology & $\begin{array}{r}\text { ADC } \\
\text { value }\end{array}$ & $\begin{array}{l}\text { ADC ratio } \\
\text { Mean } \pm S D\end{array}$ \\
\hline Medullablastoma & $0.67 \pm 0.14$ & $0.86 \pm 0.19$ \\
\hline $\begin{array}{l}\text { Pilocytic } \\
\text { astrocytoma }\end{array}$ & $1.72 \pm 0.27$ & $2.19 \pm 0.4$ \\
\hline Ependymoma & $1.09 \pm 0.14$ & $1.43 \pm 0.13$ \\
\hline BrainstemGlioma & $1.18 \pm 0.06$ & $1.64 \pm 0.08$ \\
\hline Hemangioblastoma & 1.46 & 1.87 \\
\hline $\begin{array}{r}\text { Atypical } \\
\text { Meningioma }\end{array}$ & 0.72 & 0.85 \\
\hline
\end{tabular}

with respect to $\operatorname{JPA}(n=4)$ with , the cystic component appeared hypo intense on DWIs (b1000) compared to normal appearing brain parenchyma , the solid mural nodule in JPA denoting restricted diffusion, the $A D C$ value $1.72 \pm 0.27 \times 10-3 \mathrm{~mm} 2 / \mathrm{s}$ and ADC ratio $2.19 \pm 0.4$ table $(2,3)$.Figure $(2,3)$ Regarding ependymoma $(n=3)$, the signal intensity was heterogeneous on both DWI and ADC map due to presence of solid and cystic components, the solid enhanced component displayed hypo to iso-intense on DWI and hypointense on ADC map suggesting not restricted diffusion and had $A D C$ value $1.09 \pm 0.14$ x $10-3 \mathrm{~mm} 2 / \mathrm{s}$ and ADC ratio1.43 \pm 0.13 table $(2,3)$ .Figure (2) Concerning brainstem glioma $(n=2)$ the signal intensity hypointese relative to normal brain parenchyma with ADC value $1.18 \pm 0.06 \times 10$ $3 \mathrm{~mm} 2 / \mathrm{s}$ and ADC ratio of $1.64 \pm 0.08$ on ADC map shown not restricted Hemingioblastoma $(n=1)$, solid nodule shown strong and homogenous contrast enhancement with high diffusibility and ADC value $1.46 \times 10-3 \mathrm{~mm} 2 / \mathrm{s}$ and ratio 1.87 table ( 2 ) In examination of atypical meningioma $(n=1)$ appear hyperintense in DWI to hypointense in ADC map, had $A D C$ value of $0.72 \times 10-3 \mathrm{~mm} 2 / \mathrm{s}$ and ADC ratio of 0.85 table ( 2 ) Due to limited number of cases, the three dominant categorize in this study were considered for statistical analysis There were significant difference in ADCvalue and ratio between medulloblastoma \& pilocytic astrocytoma \& significant difference in comparison between ependymoma\& pilocytic astrocytoma with $\mathrm{P}$ value $<0.05$ ) table ( 3 ). The ADC value of medulloblastoma was significantly lower than that of ependymoma \& pilocytic astrocytoma $(0.67 \pm 0.14$, vs ependymoma $1.09 \pm 0.14$ \&pilocytic astrocytoma $1.72 \pm 0.27$ in $P$ value $<0.05$ ) table (3).

Table (3): ADC value and ADC ratio of medullablastoma, JPA, ependymoma

\begin{tabular}{|c|c|c|c|}
\hline $\begin{array}{l}\text { Param } \\
\text { eter }\end{array}$ & $\begin{array}{c}\begin{array}{c}\text { Medullabl } \\
\text { astoma }\end{array} \\
\mathrm{N}=8 \\
\text { Mean } \pm \text { SD }\end{array}$ & $\begin{array}{l}\text { Pilocytic } \\
\text { astrocytoma } \\
\qquad \mathrm{N}=4 \\
\text { Mean } \pm \text { SD }\end{array}$ & $\begin{array}{c}\begin{array}{r}\text { Ependymom } \\
\text { N=3 }\end{array} \\
\text { Mean } \pm \text { SD }\end{array}$ \\
\hline $\begin{array}{l}\text { ADC } \\
\text { value }\end{array}$ & $0.67 \pm 0.14$ & $1.72 \pm 0.27^{*}$ & $\begin{array}{c}1.09 \pm 0.14^{*} \\
\dagger\end{array}$ \\
\hline $\begin{array}{l}A D C \\
\text { ratio }\end{array}$ & $0.86 \pm 0.19$ & $2.19 \pm 0.4^{*}$ & $\begin{array}{c}1.43 \pm 0.13^{*} \\
\dagger\end{array}$ \\
\hline
\end{tabular}

* Significant difference in comparison with medulloblastoma $(P$ value $<0.05)$, †Significant difference in comparison with pilocytic astrocytoma $(P$ value $<0.05)$

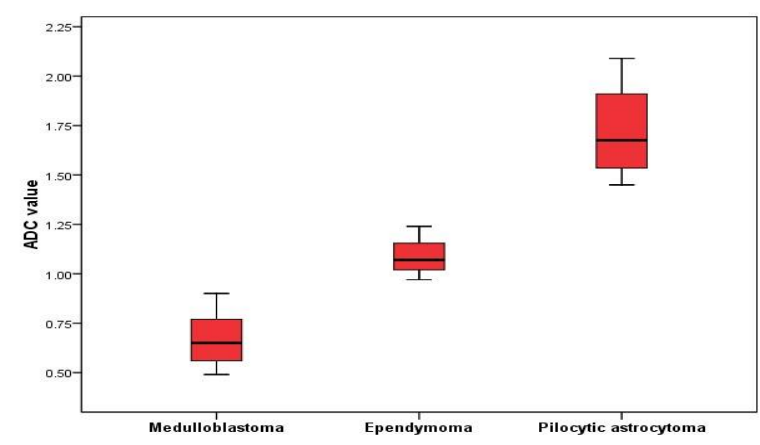

Figure( 1): Box plot of ADC value distribution in medullablastoma, ependymoma\&JPA 


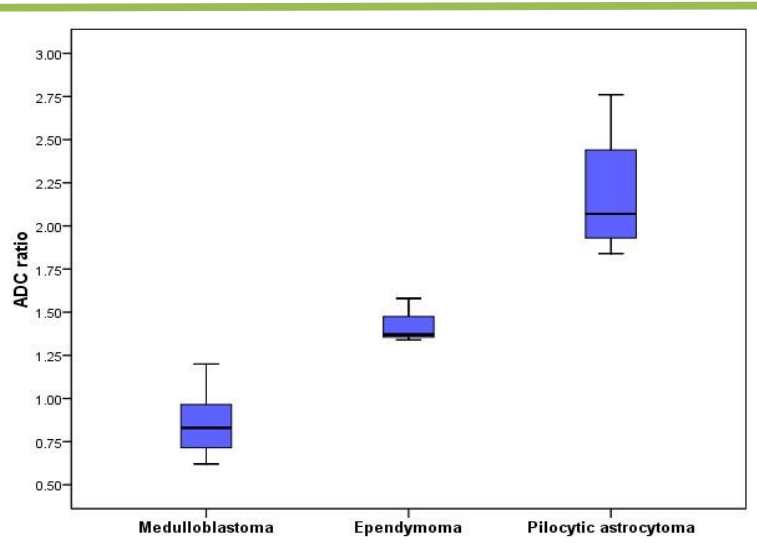

Figure ( 2 ):Box plot of ADC ratio distribution in medullablastoma, ependymoma \& JPA
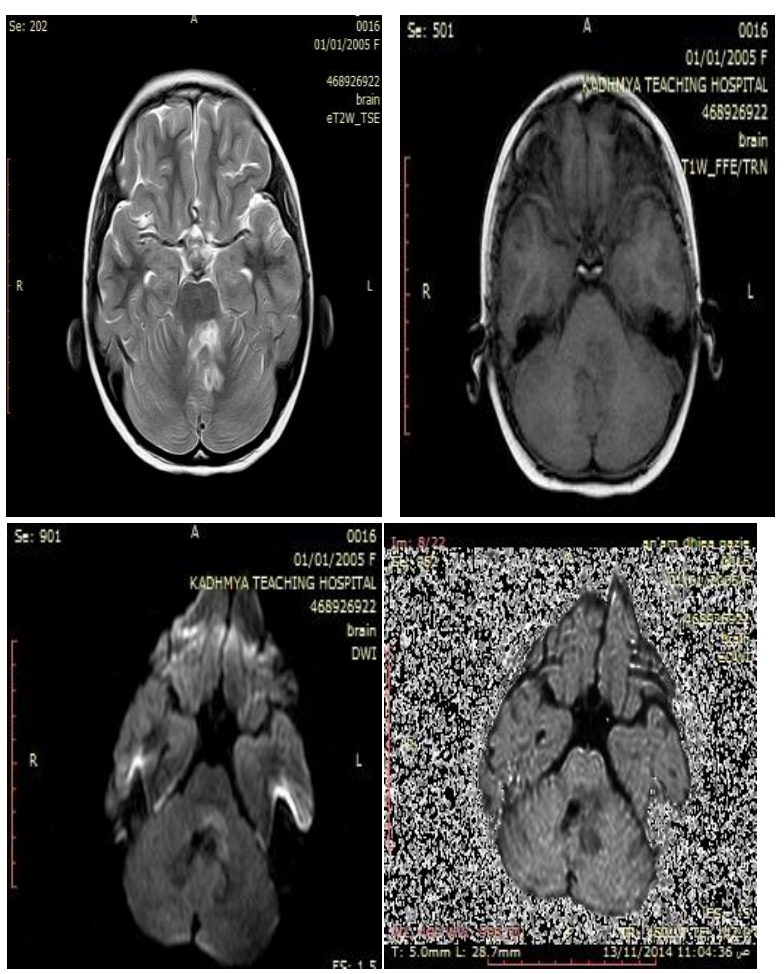

Figure (3)A-Axial T2 W show midline posterior fossa SOL of 10 year female . B-Axial T1 post Gd the SOL heterogeneous pattern of enhancement .C-axial DWI shows an intermediate signal intensity of the lesion restricted diffusion- $D$ the tumor was confirmed histopathology as ependymoma .

Discussion : conventional MRI is essential tool for diagnosis and evaluation of location ,quality ,and extend of posterior fossa tumors, but offers limited information regarding tumor grade and type .Advanced MRI techniques such as diffusion weighted imaging (DWI) may improve the specific diagnosis of brain tumors in the posterior fossa in children \& adult(22) .In our study, medulloblastoma and JPA the $A D C$ value and ratio were significantly different with no overlap. The ADC values was clearly different between medulloblastoma and ependymoma in all patients , again without overlap This finding is similar to that in a recent study by Ymasaki et al .(19), who found that $A D C$ value were retrospectively $100 \%$ accurate in differentiation between ependymomas and medulloblastoma ,the ADC of ependymoma is higher than $1.00 \times 10-3 \mathrm{~mm} \mathrm{2/s}$. The ADC of medulloblastoma is consistently lower than $1.00 \times 10-3 \mathrm{~mm} 2 / \mathrm{s}$. A number of studies reported that increasing cellularity leads to increased signal intensity on DWI and consequently hypointensity on ADC map. (Sugahara et al (10) Cheng et al (23)). The high cellularity of medulloblastoma with its densely packed cell and large nuclei is a well-known histologic feature of these tumors. $(25,26)$ which is different form the low cellularity and relatively small nuclear area typically seen in the other posterior fossa tumor types. The ADC values of JPA were also higher from ependymoma in our study .Absolute ADC values of the contrast enhancing solid tumor region in children showed that ADC values were significantly higher in pilocytic astrocytomas than in ependymomas and medulloblastomas. this finding is in concert with previous work by Cheng et al (23) who suggested that preoperative determination of the ADC of forth ventricle tumors makes the differential diagnosis possible between ependymomas and medulloblastomas .In haemangioblastoma the solid and enhancing portion appear low signal on DWI ,the $A D C$ was increased, these finding may indicate rich vascular space of haemangioblastoma, our finding is thus similar to Quadery et al (26). ADC values and ratio could prove to be reliable for distinction of other intracranial tumors, isolated analysis of diffusion properties does not provide universally reliable identification of different brain tumor types and grade ; however, this may not be clinically relevant, because diagnosis in never based on single sequence but rather on careful analysis of entire brain MR imaging study. For example, in addition to JPAs, hemangioblastoma and other posterior fossa tumors that have been found to have similar high ADC values , these neoplasms may therefore not be distinguished solely on basis of their diffusion properties Yamasaki \&Quandy et al $(19,26)$. Our results for atypical meningioma were consistent with reported in the literature Filippi et al (17). Where they found that the mean of ADC value of grad 2 meningioma range from $0.7 \pm 0.09 \times 10-3 \mathrm{~mm} 2 / \mathrm{s}$ to $0.5 \pm 0.12 \times 10-3 \mathrm{~mm} 2 / \mathrm{s}$ and the mean $A D C$ ratio range from $0.93 \pm 0.18$ to $0.85 \pm 0.15$. Based on our result, we suggest that ADC values may play potentially important role in preoperative management of children\& adult with posterior fossa tumors. If high ADC value is detected, a patient may go directly to surgery without additional imaging,assuming the he mass is pilocytic astrocytoma given that pilocytic astrocytomas are unlikely to metastasise. On the other hand, very low ADC value suggests that the tumor is medulloblastoma, so imaging of the spine is essential to exclude metastases and appropriately stage the patient. Limitation of our study include a relatively small number of patients and limited sample of pathology encountered. Conclusion The calculation of apparent diffusion coefficient value in the solid enhancing portion of tumor is a simple technique for evaluation of posterior fossa neoplasms and is useful in differentiating between the common types of 
posterior fossa tumors, namely medulloblastoma at one end and JPA and ependymoma at other end as the former has ADC of $<1$ while other tumors have $A D C$ value of $>1$. We recommend the use of diffusion weighted imaging and calculation of ADC value in cases of posterior fossa tumors as it provides additional information in characterization tumor and in pre surgical planning.

\section{References}

1. Arle JE, Morriss C, Wang ZJ, et al. Prediction of posterior fossa tumor type in children by means of magnetic resonance image properties, spectroscopy, and neural networks. J Neurosurgery 1997;86:755-61.

2. Edward escott, engen c.hin, practical differential dignosis for CT and MRI avilable in [ https/ books.google.iq/books medical] -,.2011.

3. Kristian aquiline ANCR>volme 13 number 4> ANCR.com. uk july/august2003.

4. Becker L. Pathology of pediatric brain tumors. Neuroimaging Clin N Am1999;9:671-90

5. William E. Brant, Clydea .Helms Fundamentals of diagnostic radiology, third edition ,p 48,2007.

6. Rowley HA, Grant PE, Roberts TPL. Diffusion MR imaging. Neuroimaging Clin N Am 1999;9:343-61

7. Schaefer PW, Grant PE, Gonzalez RG. Diffusionweighted MR imaging of the brain. Radiology 2000;217:331-45

8. Castillo M, Mukherji SK. Diffusion-weighted imaging in the evaluation of intracranial lesions. Semin Ultrasound CT MR 2000;21:40516

9. Stadnik TW, Chaskis C, Michotte A, et al. Diffusion-weighted MR imaging of intracerebral masses: comparison with conventional MR imaging and histologic findings. AJNR Am J Neuroradiol 2001;22:969-76

10. Sugahara $T$, Korogi $Y$, Kochi $M$, et al. Usefulness of diffusion-weighted MRI with echo-planar technique in the evaluation of cellularity of gliomas.J Magn Reson Imaging 1999;9:53-60

11. Gupta RK, Cloughesy TF, Sinha U, et al. Relationships between choline magnetic resonance spectroscopy, apparent diffusion coefficient and quantitative histopathology in human glioma. J Neurooncol 2000;50:215-26

12. Gauvain KM, McKinstry $R C$, Mukherjee $P$, et al. Evaluating pediatric brain tumor cellularity with diffusion-tensor imaging. AJR Am J Roentgenol2001;177:449-54

13. Guo AC, Cummings TJ, Dash RC, et al. Lymphomas and high-grade astrocytomas: comparison of water diffusibility and histologic characteristics. Radiology 2002;224:177-83
14. Tien RD, Felsberg GJ, Friedman $H$, et al. MR imaging of high-grade cerebral gliomas: value of diffusion-weighted echoplanar pulse sequences. AJR Am J Roentgenol 1994;162:67177

15. Kono K, Inoue $\mathrm{Y}$, Nakayama $\mathrm{K}$, et al. The role of diffusion-weighted imaging in patients with brain tumors. AJNR Am J Neuroradiol2001;22:1081-88

16. Castillo M, Smith JK, Kwock L, et al. Apparent diffusion coefficients in the evaluation of highgrade cerebral gliomas. AJNR Am J Neuroradiol2001;22:60-64

17. Christopher G. Filippi, Mark A. Edgar, Aziz M. Ulug", Joan C. Prowda, Linda A. Heier, and Robert D. Zimmerman Appearance of Meningiomas on Diffusion-weighted Images: Correlating Diffusion Constants with Histopathologic Findings AJNR Am J Neuroradiol .2001.22:65-72,

18. Bulakbasi N, Guvenc I, Onguru O, et al. The added value of the apparent diffusion coefficient calculation to magnetic resonance imaging in the differentiation and grading of malignant brain tumors. J Comput Assist Tomogr 2004;28:735-46

19. Yamasaki F, Kurisu K, Satoh K, et al. Apparent diffusion coefficient of human brain tumors at MR imaging. Radiology 2005;235:985-91

20. Calvar JA, Meli FJ, Romero C, et al. Characterization of brain tumors by MRS, DWI and $\mathrm{Ki}-67$ labeling index. J Neurooncol 2005;72:273-80

21. Kotsenas AL, Roth TC, Manness WK, et al. Abnormal diffusion-weighted MRI in medulloblastoma: does it reflect small cell histology? Pediatr Radiol1999;29:524-26

22. Krant M mastuda ,PEllen. application of new MR technique in pediatric patient magnetic Resonance imaging Clinics N August 2003;11;493-522).

23. Cheng YC Lirng JF,Chang FC et al.neurradiological findings in atypical teratoid /rhabdoid tumor of central nervous system .Acta Radiol 2005;46;89-96

24. Ellison D. Classifying the medulloblastoma: insights from morphology and molecular genetics. Neuropathol Neurobiol 2002;28:257-

25. Kleihues P, Louis DN, Scheithauer BW, et al. The WHO classification of tumors of the nervous system. J Neuropathol Exp Neurol 2002;61:21525.

26. QuandyaA,OkamotoK.DiffusionweightedMRlof,ha emangiomablastoma and other cerebellar tumors .neuroradiology 2003;45;212-19). 